\title{
Comparison Of GaAs (III-Vsemiconductors) And Si Vertical Multijunction Solar Cells, As The Converters In The Power Beaming Systems
}

\author{
Hamid Heydari ${ }^{1}$, Sabah Gaznaghi ${ }^{2}$, Muhammad Shojaei Pour ${ }^{3}$, \\ Muhammadshahraki* \\ ${ }^{I}$ Razi University, Kermanshah \\ ${ }^{2}$ Urmia University \\ ${ }^{3}$ Iran University of Science and Technology \\ *Sistan and Baluchistan University
}

\begin{abstract}
In electricity transmission systems by the laser radiation, the GaAs photovoltaic converters with more than 50\% efficiency are used. Recently the VMJ also has been studied for the monochromatic light conversion into the electricity. Here, these technologies and their application in electricity transmission systems, have been studied and evaluated. The processing method, structure and conversion efficiency have been compared. Results prove that VMJ silicon solar cells along with concentrator have the high conversion efficiency equal to $43 \%$ for the monochromatic light with $975 \mathrm{~nm}$ wavelength. Although the GaAs cells along with concentratorhave the $58.3 \%$ conversion efficiency. Considering the complexity and processing costs the silicon cells have the better situation, however if the concentratoris presented in production process, there is not considerable difference between them.
\end{abstract}

Keywords: Optical Power Transmission, GaAs, Vertical Multijunction, Diode Laser

\section{Introduction}

Optical power transmission (OPT) is a suitable method that the Copper wiringcan be replacedby the optical fibersor even the air as the medium. This replacement is justifiable because of the reasons such as short circuit danger and sparkle of power transmission lines by the copper wires or the thunderbolt clash to the power lines (Oliva, 2008).Also this replacement allows to transmit the information and energy via one signalsimultaneously. The OPT system consists of 3 part as it can be seen in figure 1 .
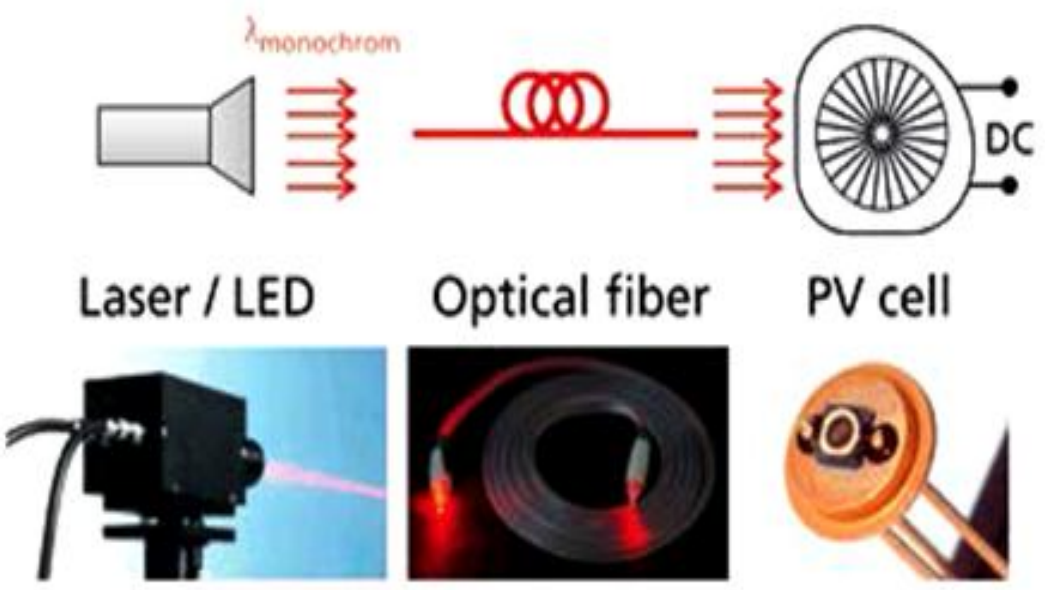

Fig1. The OPT system structure (Helmers, 2014)

The electricity can be transmitted as photons and via the optical fiber or the air. For this aim a Monochromatic light source is needed such as laser. Because the bandgap of photovoltaic converters can be set with an especial wave length, the Monochromatic light can be converted to the electricity by a solar cell with higher conversion efficiency than the solar radiation.Especially the GaAs in this domain is highly regarded by the researchers. The III-V Semiconductor's bandgap can be changed well by the elements compound. In Fraunhofer ISE, the desired wavelength have been achieved for each semiconductor via the III-V elements combining. In table 1 some of these cutoff wavelengths have been shown in $300{ }^{\circ} \mathrm{K}$. 
Table1. The III-V elements ratio in corresponding cutoff wavelength and compounds (Helmers, 2014)

\begin{tabular}{|l|l|}
\hline The element's ratio & Cutoff wavelength \\
\hline $\mathrm{Ga} 0.51 \mathrm{In} 0.49 \mathrm{P}$ & $660 \mathrm{~nm}$ \\
\hline $\mathrm{GaAs}$ & $870 \mathrm{~nm}$ \\
\hline $\mathrm{Ga} 0.83 \mathrm{In} 0.17 \mathrm{As}$ & $1050 \mathrm{~nm}$ \\
\hline $\mathrm{Ga} 0.16 \mathrm{In} 0.84 \mathrm{As} 0.31 \mathrm{P} 0.69$ & $1100 \mathrm{~nm}$ \\
\hline $\mathrm{Ga} 0.47 \mathrm{In} 0.53 \mathrm{As}$ & $1680 \mathrm{~nm}$ \\
\hline $\mathrm{GaSb}$ & $1700 \mathrm{~nm}$ \\
\hline
\end{tabular}

\section{Si And GaAs Comparison, Used In Optsystems}

The GaAs mineral structure is similar to diamond cubic crystal structure withzinc blend crystal structure. The lattice constant of GaAs and silicon are equal to $0.565 \mathrm{~nm}$ and $0.543 \mathrm{~nm}$ and theirdensity are $4.42 \times 10^{22}$ and $5.0 \times 10^{22}$ respectively (Smets, 2016).

$\mathrm{GaAs}$ is a semiconductor with direct bandgap equals to $1.42 \mathrm{eV}$ while the silicon is a Semiconductor with $1.12 \mathrm{eV}$ indirect bandgap. In optical power transmission systems the most important part is the state ofcoupling or correlation between the bandgap and laser monochromatic wavelength (Helmers, 2014).According to graph 1 , the GaAs absorption coefficient in visible and Infra-redspectrumis not only bigger than silicon but also has asteeper slope. It means that GaAs and other III-V semiconductors will be better coupledwith diode laser's monochromatic radiation $(790-840 \mathrm{~nm})$.

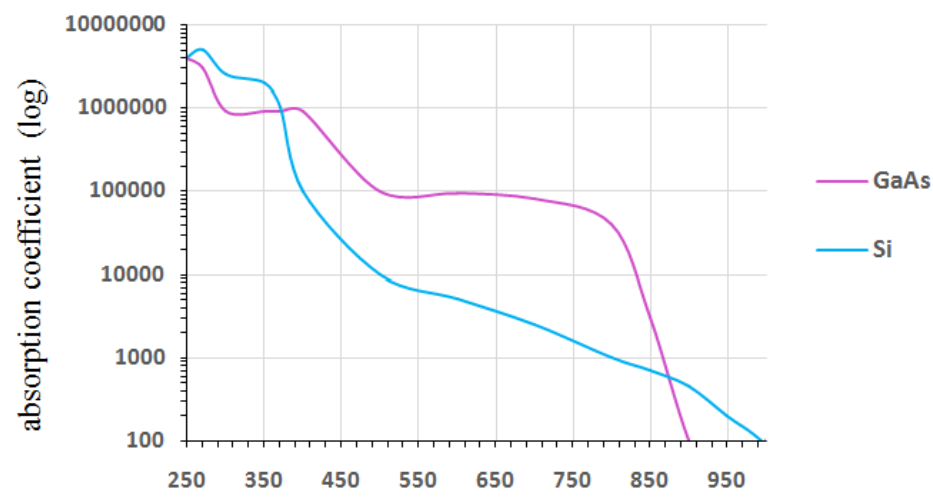

Graph 1. Comparison of the silicon and GaAs absorption coefficient

\section{The GaAs photovoltaic convertors}

The GaAs photovoltaic converters made of III-V semiconductors will be coupled well with monochromatic laser radiation, because of their bandgap structure and Adjustability.These converters have single-junction structures and especially designed for the better coupling with considered wavelength. Shan (2015) designed an optimum structure that had the $61.8 \%$ efficiency in theory at $840 \mathrm{~nm}$ wavelength (fig 2).

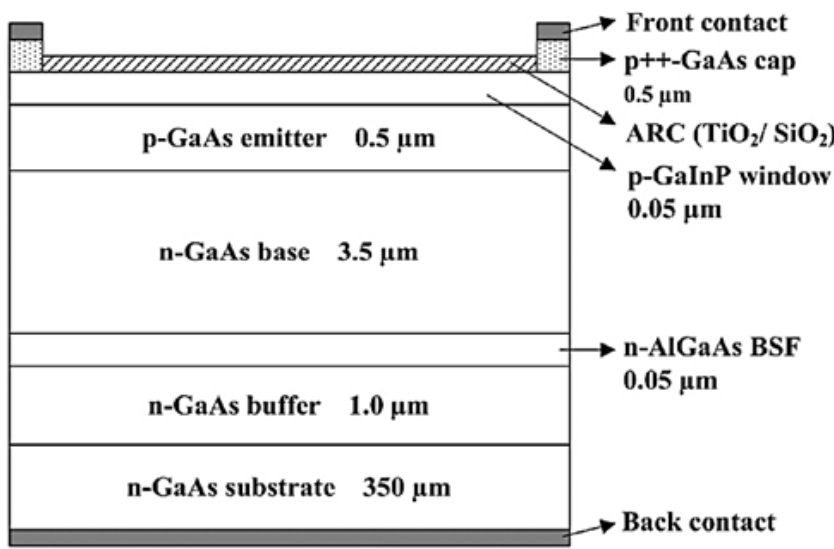

Fig2. The optimized structure of photovoltaic converters with n-AlGaAs BSF layer. (Shan, 2015)

Before the experimental works, Shan simulated an optimized model by a 1-D finite element program (PC-1D) and variable amount such as layer thickness, dopant concentration, wavelength of 790-840 nm and incident radiation equal to 0.1 to $5 \mathrm{wat} / \mathrm{cm}^{2}$. They obtained an optimum thickness of $3.5 \mu \mathrm{m}$ for base layer or nGaAs.Thinner base layer doesn't able to collect enough transmitted photons from the emitter layer and actually 
with thicker thickness will rise the resistivity of equivalent circuit series resistivity.Considering the graph 2 and 3 , the low doping decreased the open circuit voltage and Conversion efficiency consequently. On the other hand the high doping decreased the generated carrier's distribution and consequently the short circuit current.
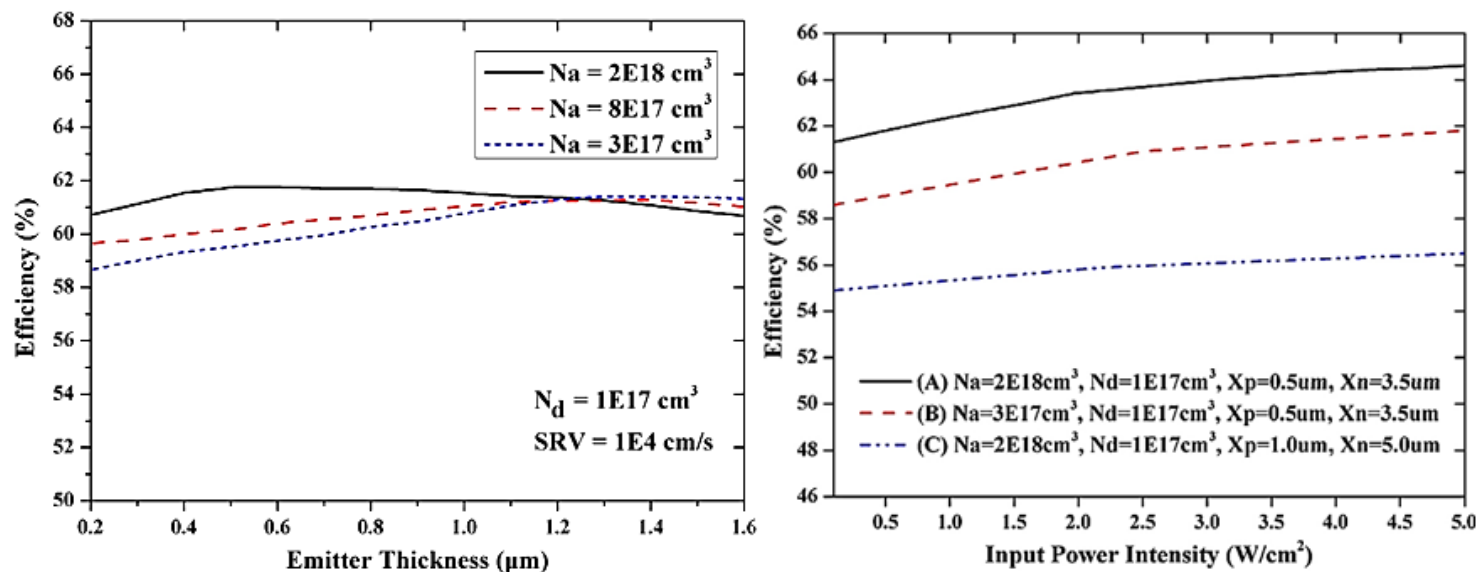

Graph2. The efficiency of GaAs photovoltaic converters against the right: laser radiation incident power and left: Emitter thickness in micron (Khvostikov et al., 2014 and Khvostikov et al., 2015).

The modeling calculations revealed that the most efficiency will be occurred that dopantconcentration in emitter and base layers are $2 \times 10^{18} \mathrm{~cm}^{3}$ and $1 \times 10^{17} \mathrm{~cm}^{3}$ respectively. Also the emitter and base layer thickness is 0.5 and $3.5 \mu \mathrm{m}$ respectively.In this situation, surface recombination velocity, SRV, has been obtained equal to $1 \times 10^{4} \mathrm{~cm} / \mathrm{s}$. finally a prototype has been provided by the MOCVD method. Table 2 shows the converter's electrical operation characteristics. The best wavelength of laser based on simulation, is $840 \mathrm{~nm}$. However in 750 to $855 \mathrm{~nm}$ spectrum more than 50\% efficiency is still accessible. Mukherjee et al (2013) studied the conversion efficiency by making of an InGaAsP/InP Multi-junction structure.

Table2. Electric properties of made prototype (Shan, 2015)

\begin{tabular}{|l|l|l|l|l|l|l|}
\hline Pin (W) & ISC (A) & VOC (V) & Imax (A) & Vmax (V) & Fill Factor & $(\%) \eta$ \\
\hline 0.5 & 0.272 & 1.122 & 0.262 & 0.991 & 0.851 & 51.93 \\
\hline 3 & 1.666 & 1.127 & 1.594 & 0.994 & 0.844 & 52.81 \\
\hline 5 & 2.793 & 1.135 & 2.653 & 1.001 & 0.840 & 53.23 \\
\hline
\end{tabular}

Above values can be extracted from the I-V curve. Khvostikov et al (2015) designed a photovoltaic converter as AlGaAs/GaAs and provided it by the Liquid Phase Epitaxy (LPE) method.This converter has been designed and produced for the high radiation power Conversion in wavelength of $809 \mathrm{~nm}$ (fig 3).

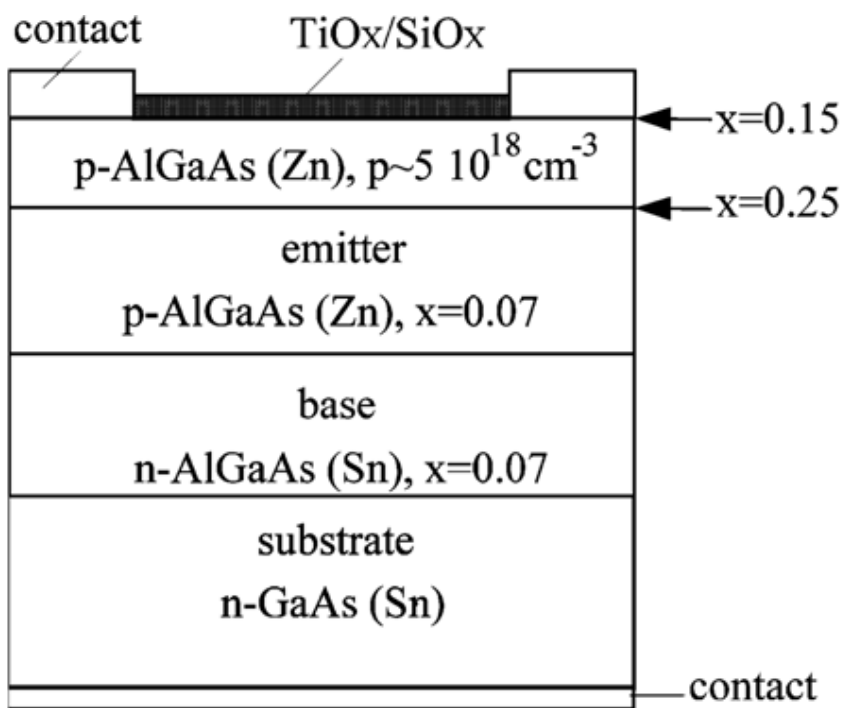

Fig3. Photovoltaic converter's structure for coupling with high power laser with wavelength of $809 \mathrm{~nm}$. 
Indeed the main work of Khvostikov et al was determining of photosensitivity in various layers in wavelength of $809 \mathrm{~nm}$. Here the $\mathrm{p}$ - $\mathrm{n}$ junction is designed as $\mathrm{Al}_{\mathbf{x}} \mathrm{Ga}_{\mathbf{1 - x}} \mathrm{As}$ that $\mathrm{x}$ is equal to 0.07. Unlike the conventional multijunction structures, there is no need for the photosensitivity towards the short wavelength in upper cell or window layer. Considering the figure 3 it is obvious that the aluminum density in window layer, which has a good photosensitivity to the short wavelength, is decreased from 0.25 to 0.15 by moving from down to the front contacts.
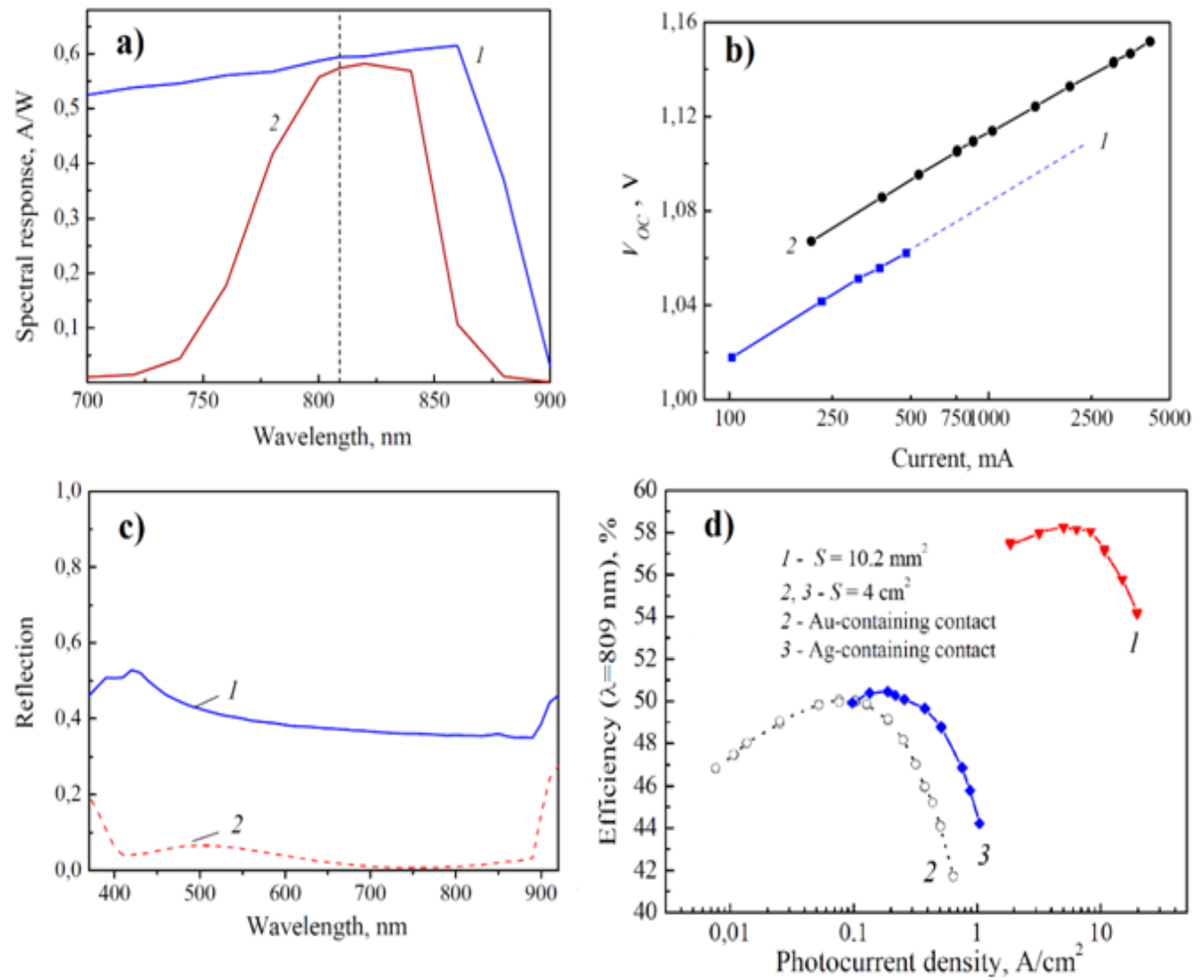

Graph3. a) Spectral response of conventional PV converters (1) and Laser PV converters (2). b)Comparison of the open circuit voltage in conventional PV converters (1) and Laser PV converters which is made by LPE method (2). c) Reflection coefficient for converters with (2) and without (1) TiO2/SiO2 ARC. d) Conversion efficiency for Laser PV converters with, smaller area (1), large surface area and gold contacts (2) large surface area and silver contacts (3). (Khvostikov et al., 2014 and Khvostikov et al., 2015).

One of the Aluminum low density benefits at junctions, shows the junction situation improvement and decreasing the amount of surface recombination (Khvostikov et al., 2014 and Khvostikov et al., 2015). Similar to Shan et al, here the $\mathrm{TiO} 2 / \mathrm{SiO} 2$ anti-reflectioncoating (ARC) has been used for the diminishingoptical losses.Khvostikov et al investigated performance differences, especiallyin spectral response and open circuit voltage, betweensolar cells and laser photovoltaic converters, which is made via LPE method.

Conversion efficiency related to laser photovoltaic converter in graph 3-d shows that depend on cell surface area the efficiency changes from $50 \%$ to $58.3 \%$. Decreasing the cell surface area the conversion efficiency has been increased. This efficiency increasing has been attributed to the series resistance decreasing and increasing of cell equivalent shunt resistance and also the heat loss decreasing. Also the Ohmic resistance reduction at metal-semiconductor junction due to material changes and thickness increasing, caused the improvement output properties such as output current density.

\section{The verticalmultijunction Si photovoltaic converters for the high intensity radiation}

The structure of VMJ silicon photovoltaic converters have the different structure than the conventional structures. These cells have been designed for the intense incident radiation and especially monochromatic ones. As it can be seen in figure 4 the structure consists of many series junctions as it can provide low resistivity. On the other hand the series cell junctions present the high output voltage and low output current that made it suitable for using under concentrated radiations. Indeed one of the most considerable benefits of this technology is the high produced voltage (Nayfeh, 2008). 


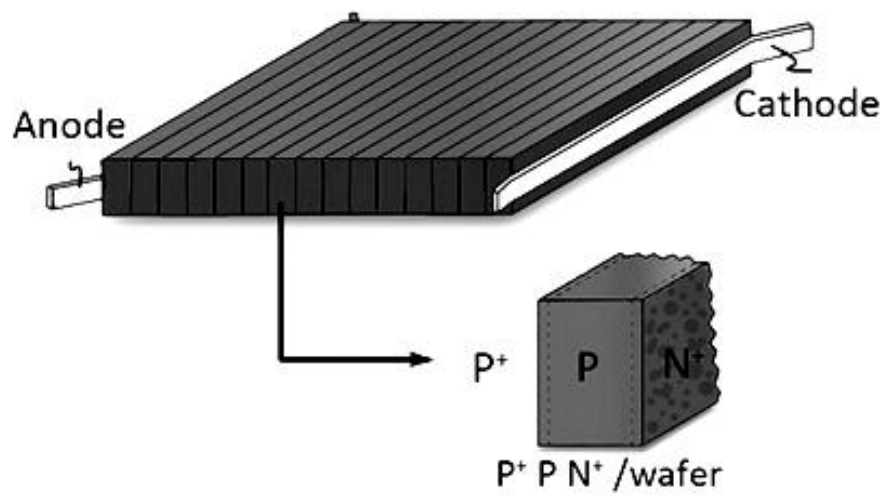

Fig4. VMJ converter structure for the monochromatic and concentrated radiations (Nayfeh, 2008)

Nayfeh et al used these converters for the airplane electric engine. They achieved the conversion efficiency up to $44.39 \%$ under the 200 wat radiation and $975 \mathrm{~nm}$ wavelength.But it can be seen that heat factor has more devastating impact on conversion efficiency. As for each linear $10^{\circ} \mathrm{C}$ the conversion efficiency will reduced $2.5 \%$. This problem will be more critical in intense and concentrated radiation. Therefore wide researches have been done about this problem.

Tung et al suggested a solution for this problem, usingtwo layersof Boron-Nitride and one layer of Aluminum-Nitride between them. This structure as the Thermal Epoxy, upon to figure 5, well conducts the heat from a direction of VMJ silicon cells toward the copper pipe units. This process improves the VMJ cells operation.

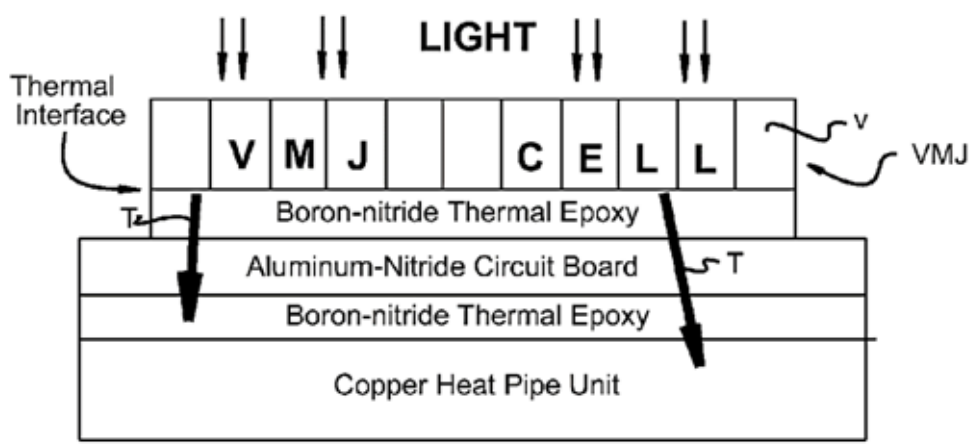

Fig5. The structure of VMJ silicon photovoltaic which is equipped with heat-conducting layers. Arrows show the flow of heat transfer (Tung, 2016).

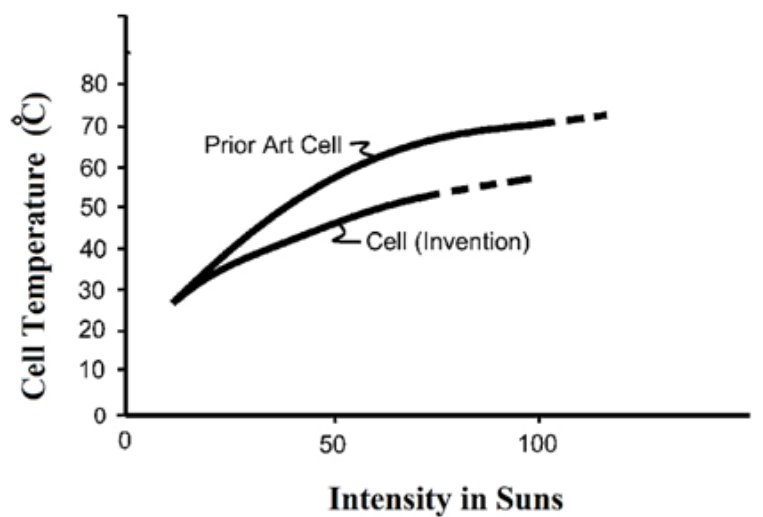

Graph4. The temperature of VMJ cell operation as a function of incent radiation intensity

As it can be seen in graph 4, in comparing produced prototype with solar cells without this technology, the rising of temperature along with increasing in radiation intensity has a gradualslope (Tung, 2016).Perales et al (2016) designed a setup for the VMJ cells, and achieved to $43 \%$ efficiency and 31 volts density per centimeter under the laser radiation equal to $1 \mathrm{~kW}$ attwithwavelength of $976 \mathrm{~nm}$. 


\section{Conclusion}

The OPT systems generally use of GaAs photovoltaic converters. But recently the VMJ photovoltaic converters also qualified for using in this domain. In all studies about the GaAs converters at first an optimum structure has been designed and simulated and after that it has been made. Most of simulations has been done in one dimension and therefore there are differencesin operational results. The GaAs cells have higher conversion efficiency, because of their better couplingwith monochromatic radiations and thermal behavior. But in situations that the higher voltage is needed, (more than 20 volts) the cells should be linked as series form. VMJ converters for this aim seems to be suitable but indeed they are sufferingfrom power lossesdue to the temperature rising, however, Tung et al solved this problem, using the heat transmission copper pipes.

\section{References}

[1]. Smets, Arno. et al. (2016). Solar Energy: The Physics and Engineerings of Photovoltaic Conversion Technologies and Systems. UIT Cambridge Ltd. ISBN-13. 978-1906806325

[2]. E. Oliva, F. Dimroth, GaAs converters for high power densities of laser illumination, Prog. Photovolt.: Res. Appl. 16 (2008) 289295

[3]. Shan, Tiqiang and Qi, Xinglin. (2015). Design and optimization of GaAs photovoltaic converter for laser power Beaming. Infrared Physics \& Technology 71 (2015) 144-150

[4]. Helmers, Henning et al. (2014). Photovoltaic Cells for Optical Power and Data Transmission. European Telemetry and Test Conference, Nurnbergm Fraunhofer ISE, June 3, 2014.

[5]. Mukherjee, J et al. (2013). Efficiency limits of laser power converters for optical power transfer applications. J. Phys. D: Appl. Phys. 46 (2013) 264006 (6pp)

[6]. Khvostikov, Vladimir et al. (2015). AlGaAs Converters and Arrays For Laser Power Beaming. AIP Conference Proceedings 1679, 130002 (2015); doi: 10.1063/1.4931562

[7]. Khvostikov, Vladimir et al. (2014). "AlGaAs/GaAs photovoltaic converters for high power narrowband radiation" in 10th International Conference on Concentrator Photovoltaic Systems - 2014, AIP Conference Proceedings 1616, edited by G. S. Kinsey et al. (American Institute of Physics, Melville, NY, 2014), pp.21-24

[8]. Tung, Chiun-Yen et al. (2016). Direct Thermal Path Heat Sinking Using Fins Formed From Energy Conversion Device Components, Including Subcomponents of Vertical Multijunction Photovoltaic Receivers Used For High Intensity Beaming and Wireless Power Transmission. Patent Application Publication. Pub. No.: US2016/0005902 A1

[9]. Nayfeh, Taysir H. et al. (2008). High Intensity Laser Power Beaming Reciever for Space and Terrestrial Apllication. Patent Application Publication. Pub. No.: US 2008/0245930 A1

[10]. Perales, Mico et al. (2016). Characterization of high performance silicon-based VMJ PV cells for laser power transmission applications. High-Power Diode Laser Technology and Applications XIV. Proc. of SPIE Vol. 9733 97330U-1 